\title{
Frequency Adjusting Technique for Transient Noise Analysis of Nonlinear Integrated Circuits
}

\author{
M.M. Gourary, S.G. Rusakov, S.L. Ulyanov, M.M. Zharov \\ Institute for Design Problems in Microelectronics of Russian Academy of Sciences, ippm@ippm.ru
}

\begin{abstract}
The paper discusses the nonlinear noise simulation problems in integrated circuits. The method of automatic varying the number of harmonics is presented to reduce computational efforts of transient noise simulation techniques based on spectral decomposition of stochastic process. The adaptive procedure provides the noise simulation of nonlinear circuits without need to specify number of noise harmonics before the analysis.
\end{abstract}

Keywords - noise simulation, time-varying models, transient noise analysis, varying the number of harmonics.

\section{INTRODUCTION}

The most part of standard SPICE-like circuit simulators use computational procedures for small signal AC noise and also periodic steady state noise analysis. The mode for nonlinear transient noise analysis is desired in many cases but it is not provided as a rule. The main reason is connected with very high time consumption to solve the nonlinear noise simulation problems. Thus, the reducing of computational efforts is the major research topic to develop efficient numerical noise analysis procedures. Some positive results were obtained in this research direction [1$10]$.

Enhancing the efficiency of numerical methods and tools to estimate noise characteristics of nonlinear circuits still is an important issue for practical problems of RF design $[1,3,5]$. This paper discusses one more possible resource to reduce computational costs of noise simulation of nonlinear circuits.

The traditional AC noise analysis is used for determining noise characteristics of analog circuits which operate in small signal conditions. The analysis is based on stationary noise source models and the theory of linear time-invariant systems. It provides the probabilistic characterization in the form of output spectral densities at the frequency points of interest. Circuits which present large signal variations in their operation require timevarying noise simulation because the time-invariant assumption is no longer valid.

Some non-Monte Carlo methods of transient noise analysis have been developed both for periodic $[3,5,7,8]$ and arbitrary excitation $[1,2,4,9]$. The numerical methods $[8,9]$ essentially exploit the spectral decomposition of the stationary stochastic process.

In this case the dimension of the problem is determined by the frequency region and the number of frequency points in the decomposition. This number of frequency points is the parameter of the analysis. This parameter must be specified before analysis. The overestimated number of frequencies leads to tremendous computational efforts while the small value does not provide the desired accuracy of the analysis. Thus there is a conflict between desired accuracy and computational efforts. For this reason it is desired to determine the number of frequency points in automatic manner. Moreover such an adaptive numerical procedure provides flexible transient noise analysis by taking into account the variation of nonlinear circuit frequency properties during transient behavior.

The contribution of this paper is to introduce a technique for automatic varying the number of frequencies for the spectral decomposition to provide desired accuracy. Section 2 briefly describes the basic method of transient noise analysis. Section 3 presents the new technique of frequency adjusting for transient noise analysis and section 4 contains some numerical results.

\section{Computational Method}

The method is based on the presentation of a circuit as linear time-varying (LTV) system and uses the spectral decomposition of noise stochastic process $[9,12,13]$.

Assuming the charge oriented model and modified nodal analysis (MNA) the nonlinear circuit can be described by the following equation:

$$
\dot{q}(x(t))+i(x(t))+b(t)+A u(t)=0
$$

where $q(x), i(x)$ are vectors of node charges or fluxes and node currents, $b(t), u(t)$ are vectors of large signals and noise sources respectively (the $N$ by $K$ matrix $A$ reflects the connections of noise sources, $K$ is the total number of noise sources in the circuit). The vector $x(t)$ includes node voltages and some branch currents. Assuming the contribution of the noise sources is small, the linearization of (1) about the large-signal noise-free solution yields the following equation with respect to the vector of noise response $y(t)$ :

$$
\frac{d}{d t}(C y(t))+G y(t)+A u(t)=0
$$

where $\mathrm{C}$ and $\mathrm{G}$ are capacitance and conductance matrix respectively.

The expression (2) is a linear differential system with time-varying coefficients and therefore the overall noise response can be computed as the superposition of noise responses to every noise source: 


$$
y(t)=\sum_{k=1}^{K} y^{(k)}(t)
$$

Transient noise analysis corresponds to the nonstationary stochastic process and the modulated stationary noise models have been proposed to use in the following general form $[2,7]$ :

$$
u(t)=m(t) u_{s}(t)
$$

where $u(t)$ is the modulated noise source, $u_{s}(t)$ is the stationary noise source and $m(t)$ is the deterministic modulation. Note that sources $u_{s}(t)$ reflect thermal, shot and flicker noise [14] specified by spectral densities.

Using the spectral decomposition of zero-mean stationary stochastic process $u_{s}(t)$ the noise models can be represented in the form:

where

$$
u(t)=\sum_{l=1}^{L} \xi_{l} s_{u}\left(\omega_{l}, t\right) e^{j \omega_{l} t}
$$

$$
\xi_{l}=\frac{\xi_{l}}{\sqrt{s_{u_{s}}\left(\omega_{l}\right)}}, s_{u}\left(\omega_{l}, t\right)=m(t) \sqrt{s_{u_{s}}\left(\omega_{l}\right)},
$$

$\xi_{l}$ are noncorrelated random values with variance $s_{u_{s}}\left(\omega_{l}\right) \Delta \omega_{l}, s_{u_{s}}\left(\omega_{l}\right)$ is the spectral density of $u_{s}(t)$ and $L$ is the number of harmonics in the spectral decomposition.

Substituting (5) into (2) yields

$$
\begin{gathered}
\frac{d}{d t}\left(C y^{(k)}(t)\right)+G y^{(k)}(t)+ \\
a \sum_{l=1}^{L} \xi_{l} s_{u}^{(k)}\left(\omega_{l}, t\right) e^{j \omega_{l} t}=0
\end{gathered}
$$

where $a$ is $k$-th column of matrix $A$.

Because equation (6) is a linear differential equation with time-varying coefficients, each $k$-th response is the linear combination of responses with the coefficients $\xi_{l}$ at various frequencies:

$$
\begin{gathered}
y(t)=\sum_{k=1}^{K} y^{(k)}(t)= \\
\sum_{k=1}^{K} \sum_{i=1}^{L} \xi_{l} y^{(k)}(t)\left(\omega_{l}, t\right)
\end{gathered}
$$

Finally using the substitution

$$
y^{(k)}(t)\left(\omega_{l}, t\right)=z^{(k)}\left(\omega_{l}, t\right) e^{j \omega_{l} t}
$$
[9]:

the following linear differential equations are obtained

$$
\underset{\substack{d t \\ k=1,2, \ldots, K, l=1,2, \ldots, L}}{\left.\frac{d}{(k)}\left(\omega_{l}, t\right)\right)+}
$$

The known integration techniques can be applied to solve (9). The initial conditions are obtained from the expression

$$
\dot{Z}^{(k)}\left(\omega_{l}, t_{0}\right)=0
$$

After solving the equation (9) with respect to the envelop variable $z^{(k)}\left(\omega_{l}, t\right)$ the complete probabilistic characterization of a circuit (variance, autocorrelation, correlation and covariation) can be computed. For example, the variance is computed by the following expression:

$$
V_{\text {node }}=\int_{\omega_{\min }}^{\omega_{\max }} \sum_{k=1}^{K}\left|z_{\text {node }}^{(k)}\left(\omega_{l}, t\right)\right|^{2} d \omega
$$

The error of the method is determined by the error of numerical integration of (9) and the error due to the truncated set of harmonics in the spectral decomposition (5). To reduce the last error more harmonics have to be taken into account that increase the computational efforts. Therefore a compromise is required. Besides, often the number of harmonics is not known a priori and only the range of frequencies of interest can be estimated.

\section{FRequency AdJusting Method ApPROACH}

Our goal is to develop a method for determining the number of harmonics in (5) providing a given variance error at each time point while the transient noise analysis. The error estimates are obtained by comparing the results of integration at the boundary frequency points of intervals of single and double size. Frequency points are then inserted or deleted to preserve the desired error.

The calculation of variance (11) leads to an estimation at each time point the following integral in the frequency range $\left[\omega_{n}, \omega_{n+1}\right]$ :

$$
I_{n, n+1}=\int_{\omega_{n}}^{\omega_{n+1}} f(\omega) d \omega
$$

where $f(\omega)$ is the piecewise linear approximation of the integrand in (11), $I_{n, n+1}$ - is the estimate of the integral in the range $\left[\omega_{n}, \omega_{n+1}\right]$.

To evaluate the approximation errors at frequency intervals we can consider the integrand values in three neighboring points $\left(\omega_{m}, \omega_{k}, \omega_{n}\right)$. This is illustrated in the fig. 1 . The solid lines correspond to the approximation and dash line corresponds to the real function.

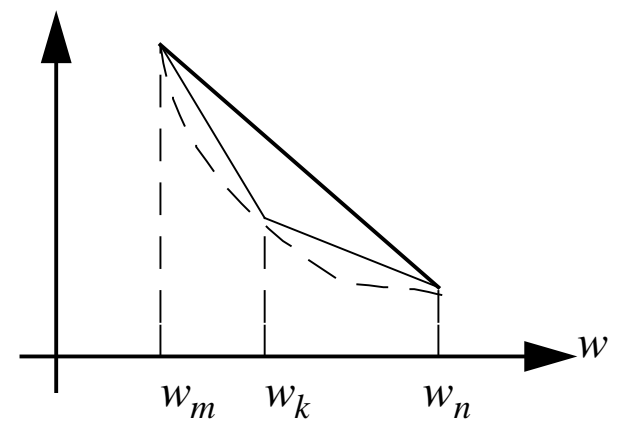

Fig. 1. Illustration of the integrand segment in (12) (dashed line) with two piecewise linear approximations (solid lines)

Let $E_{m, n}^{(m, k, n)}, E_{m, k}^{(m, k, n)}, E_{k, n}^{(m, k, n)}$ be approximation errors at the intervals $\left[\omega_{m}, \omega_{n}\right],\left[\omega_{m}, \omega_{k}\right],\left[\omega \mathrm{k}, \omega_{n}\right]$ obtained by integrals evaluations at points $\left(\omega_{m}, \omega_{k}, \omega_{n}\right)$.

We assume the approximation error to be square dependent on the length of the interval

$$
E_{i, j}=c \cdot \omega^{2},
$$


where $c$ is the coefficient defined by the polynomial approximation of the curve in Fig. 1 by three values in points $\left(\omega_{m}, \omega_{k}, \omega_{n}\right)$.

Then the following expression for current error is true:

$$
E_{m, n}^{(m, k, n)}=E_{m, n}^{m, k, n}+E_{m, n}^{m, k, n}+I_{m, k}+I_{k, n}-I_{m, n}
$$

After finding the value $c$ for the given three points and substituting (13) into (14) we can obtain errors in subintervals $\left[\omega_{m}, \omega_{k}\right]$ and $\left[\omega_{k}, \omega_{n}\right]$ :

$$
\begin{aligned}
& E_{m, k}^{(m, k, n)}=\frac{\omega_{m, k}}{2 \omega_{k, n}} \cdot I_{m, k}+I_{k, n}-I_{m, n} . \\
& E_{k, n}^{(m, k, n)}=\frac{\omega_{k, n}}{2 \omega_{m, k}} \cdot I_{m, k}+I_{k, n}-I_{m, n} .
\end{aligned}
$$

To determine the error at the interval $\left[\omega_{n}, \omega_{n+1}\right]$ the intermediate intervals specified by the points $\left(\omega_{n-1}, \omega_{n}\right.$, $\left.\omega_{n+1}\right)$ and $\left(\omega_{n}, \omega_{n+1}, \omega_{n+2}\right)$ are considered. Using (16) we can obtain from $\left(\omega_{n-1}, \omega_{n}, \omega_{n+1}\right)$ the first estimate of error $E_{n, n+1}^{(n-1, n, n+1)}$ at interval $\left[\omega_{n}, \omega_{n+1}\right]$. From points $\left(\omega_{n}\right.$, $\left.\omega_{n+1}, \omega_{n+2}\right)$ we can obtain using (15) the second estimate of error $E_{n, n+1}^{(n, n+1, n+2)}$ at interval $\left[\omega_{n}, \omega_{n+1}\right]$. Then the final error $E_{n, n+1}$ at interval $\left[\omega_{n}, \omega_{n+1}\right]$ is defined as maximum from two values: $E_{n, n+1}^{(n-1, n, n+1)}, E_{n, n+1}^{(n, n+1, n+2)}$.

If a new point is inserted in the middle of the interval $\left[\omega_{n}, \omega_{n+1}\right]$ then the error is decreased by the value:

$$
\Delta_{n}=E_{n, n+1} / 2 \text {. }
$$

Now we shall obtain the estimate of error in case of removing point $\omega_{n}$.

Considering points $\left(\omega_{n-1}, \omega_{n}, \omega_{n+1}\right)$ we obtain the estimate of error at interval $\left[\omega_{n-1}, \omega_{n+1}\right]$ before removing $\omega_{n}$ as:

$$
E_{n-1, n+1}^{\text {old }}=E_{n-1, n}^{(n-1, n, n+1)}+E_{n, n+1}^{(n-1, n, n+1)} .
$$

Considering points $\left(\omega_{n-1}, \omega_{n}, \omega_{n+1}\right)$ and $\left(\omega_{n-1}, \omega_{n+1}\right.$, $\left.\omega_{n+2}\right)$ we can obtain from (15) and (16) two estimates of error at interval $\left[\omega_{n-1}, \omega_{n+1}\right]$ after removing $\omega_{n}$ : $E_{n-1, n+1}^{(n-2, n-1, n+1)}$ and $E_{n-1, n+1}^{(n-1, n-1, n+2)}$. The resulting $n-1, n+1$ error $E_{n-1, n+1}^{n e w}$ at interval $\left[\omega_{n-1}, \omega_{n+1}\right]$ can be defined as the maximum of obtained two values. The increment of the error after removing $\omega_{n}$ is presented as:

$$
\delta_{n}=\left|E_{n-1, n+1}^{\text {new }}-E_{n-1, n+1}^{\text {old }}\right| \text {. }
$$

Thus, the following steps determine the proposed frequency adjustment method:

1) inserting step: if the total current error exceeds the specified error tolerance then

- the frequency interval with maximal value $\Delta_{n}$ (17) is determined and a new frequency point is inserted.

2) removing step: if the total current error is significantly less than the specified error tolerance then
- the frequency point $\omega_{n}$ for which the error with the increment $\delta_{n}$ (19) remains less than error tolerance is determined and this frequency point is removed.

\section{EXPERIMENTAL RESULTS}

Two simulation examples are given below that illustrate computational efficiency of the suggested technique.

The first example demonstrates the effect of number of frequencies on error of variance. The behavior of operational amplifier in the regime of class $\mathrm{C}$ was simulated in this case. Fig. 2 shows the root mean square (rms) value of output noise. Curve 1 corresponds to fixed 5 harmonics per decade. Curve 2 is obtained by using of frequency adjusting method with 5 harmonics initially specified. In this case the number of harmonics is varied during integration process from 8 to 64 per decade. Note the difference between curves 1 and 2. For comparison the curve 3 is presented that corresponds to 100 harmonics per decade. Curves 2 and 3 are in good agreement. Note that good accuracy for curve 2 is obtained with essentially less number of harmonics due to better distribution of frequencies.

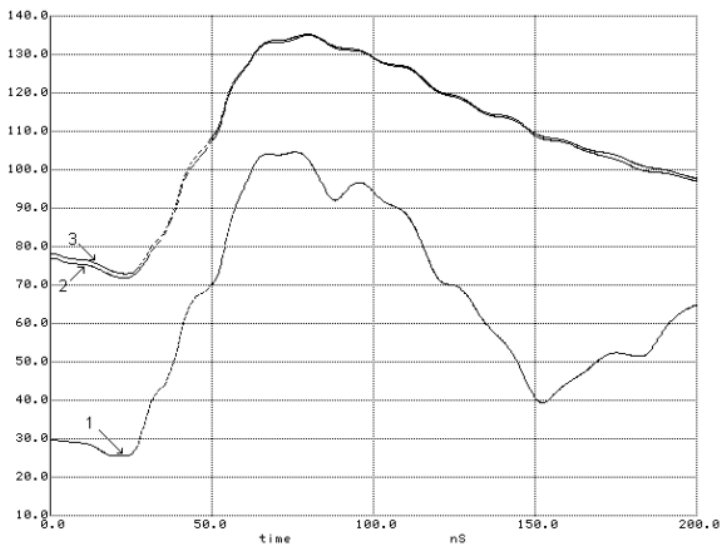

Fig. 2. RMS value of amplifier output noise as a function of time. Curve 1 is computed with fixed five harmonics per decade; curve 2 is computed with automatic adjustment of the number of harmonics; curve 3 uses fixed one hundred harmonics per decade

The computation of output noise characteristics for opamp $\mu \mathrm{a}-741$ [14] working in nonlinear regime is the second simulation example. The suggested adjusting procedure provides varying the number of harmonics from 5 to 30 per decade. Fig. 3 (curves 2, 3, 4) shows the rms value of output noise computed with different fixed numbers of frequencies, respectively 10, 20 and 30 harmonics per decade. Curve 1 corresponds to the computation using the frequency adjusting procedure. Note that curves 1 and 4 are in a good agreement. The average number of harmonics in adjusting process is 18 harmonics per decade. The acceleration of simulation by 1.8 times was achieved in comparison with 30 fixed harmonics version. 


\section{REFERENCES}

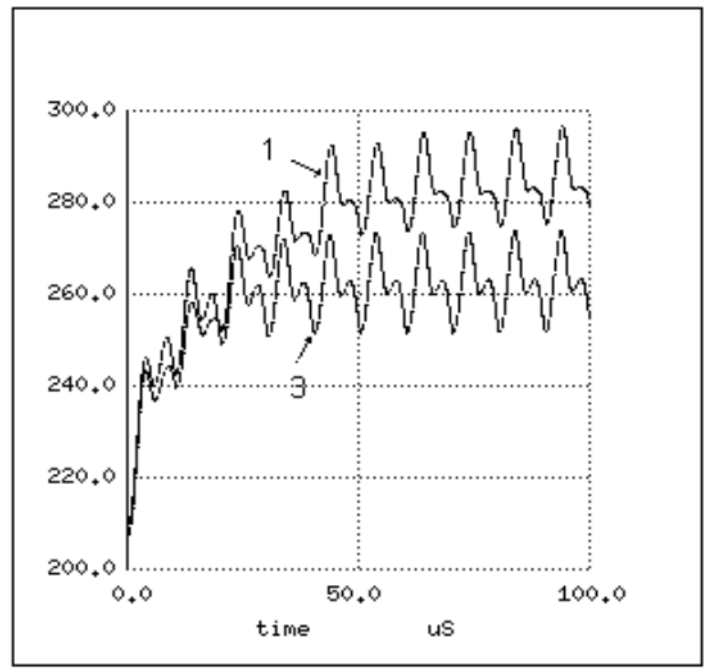

a)

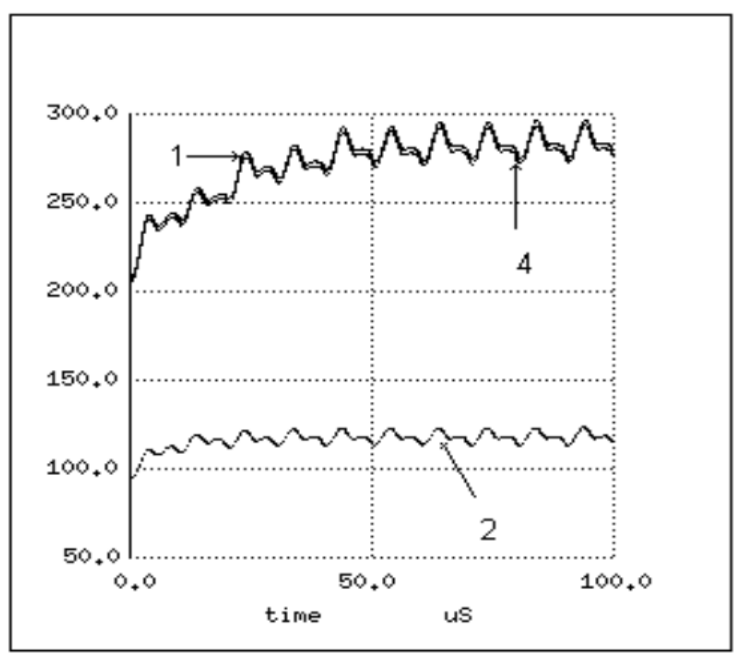

b)

Fig. 3. RMS value of ua741 opamp output noise as a function of time. Curve 1 is calculation with automatic adjustment of the number of harmonics; curves 2, 3, 4 are computed with fixed 10, 20 and 30 harmonics per decade respectively. Curves 1 and 3 are given in fig.3,b with an enlarged scale.

\section{CONCLUSION}

The proposed adaptive method performs automatic varying the number of noise harmonics during integration process in accordance with predetermined error tolerance. The adjusting algorithm is implemented in Spice-like simulator. It allows to users to eliminate the strong requirement connected with a priory specifying number of noise harmonics. Due to automatic varying the number of harmonics the method provides reducing the time spents in noise simulation of nonlinear circuits.
[1] Rizzoli V., Mastri F., Masotti D.. General noise analysis of nonlinear microwave circuits by the piecewise harmonicbalance technique. IEEE Trans. Microwave Theory Tech., 1994. vol. 42. pp. 807-819.

[2] Demir A., Liu E., Sangiovanni-Vincentelli A. Time-domain non Monte-Carlo noise simulation for nonlinear dynamic circuits with arbitrary excitations. IEEE Trans. on Computer-Aided Design, 1996. vol. 15, pp. 493-505.

[3] Roychowdhury J., Demir A. Estimating Noise in RF Systems. In Proc. ACM ICCAD. 1998. pp.199-202.

[4] Bolcato P. Poujois R. A new approach for noise simulation in transient analysis. In Proc. Int. Symp. Circuits Syst., 1992. pp. 887-890.

[5] Kundert K.S. Introduction to RF Simulation and its Application. IEEE J. of Solid State Circuits, 1999. vol.34. No. 9, pp. 1298-1319.

[6] Telichevesky R., Kundert K., White J. Efficient AC and Noise Analysis of Two-Tone RF Circuits. In Proc. IEEE DAC. 1996. pp. 292-297.

[7] Roychowdhury J., Long D., Feldmann P. Cyclostationary Noise Analysis of Large RF Circuits with Multitone Excitations. IEEE J. of Solid-State Circuits, 1998. vol. 33, No. 3, pp. 324-336.

[8] Okumura M., Tanimoto H., Itakura T., Sugawara T. Numerical Noise Analysis for Nonlinear Circuits with a Periodic Large Signal Excitation Including Cyclostationary Noise Sources. IEEE Trans. on Circuits and Systems - I, 1993. vol. 40. No. 9. pp. 581-590.

[9] Gourary M. M., Rusakov S. G., Ulyanov S. L., Zharov M. M., and. Mulvaney B. J. A New Numerical Method for Transient Noise Analysis. In Proc. ASP-DAC. 1999. pp.165-168. DOI: 10.1109/ASPDAC.1999.759986.

[10] Denk G., Romisch W., Sickenberger T., and Winkler R. Efficient transient noise analysis in circuit simulation. In Proceedings in Applied Mathematics and Mechanics. 2006. vol. 6, pp. 55-58.

[11] Gong Fang, Yu Hao, He Lei. Fast Non-Monte-Carlo Transient Noise Analysis for High-Precision Analog/RF Circuits by Stochastic Orthogonal Polynomials. In Proceedings of the 48th Design Automation Conf. 2011. pp. 298-303.

[12] Gourary M. M., Rusakov S. G., Ulyanov S. L., Zharov M.M., Gullapalli K. and Mulvaney B. J. A New Approach for Computation of Timing Jitter in PLL. In Proc. DATE, 2000. pp.345-349

[13] Gourary M. M., Rusakov S. G., Ulyanov S. L., Zharov M. M., Gullapalli K. and Mulvaney B. J. Approximation Approach for Timing Jitter Characterization in Circuit Simulators. In Proc. DATE, 2003. pp.156-161. DOI: 10.1109/DATE.2003.1253602.

[14] Gray P., Meyer R. Analysis and Design of Analog Integrated Circuits, John Wiley \& Sons, 1984. 